\title{
HIV—testing, new diagnoses, management and PEPSE \\ P1 DOES SIMPLE CORRESPONDENCE AND FEEDBACK IMPROVE HIV TESTING RATES IN PRIMARY CARE IN A LOW PREVALENCE AREA?
}

doi:10.1136/sextrans-2012-050601c.1

\author{
L Haddon, ${ }^{*} \mathrm{R}$ Bendall, K Pollard. Royal Cornwall Hospital NHS Trust, Truro, \\ Cornwall, UK
}

Background Delayed diagnosis of HIV is a problem in both high and low prevalence areas. In Cornwall, the relatively low prevalence means that most general practitioners (GPs) have little or no experience in managing patients with HIV infection; they may be unfamiliar with indicator conditions and thus be unlikely to offer an HIV test.

Aims To see if simple correspondence and feedback regarding testing rates can lead to changes in practice.

Methods We compared testing rates before and after the intervention which comprised of a letter to all individual GPs attached to a PCT weekly communication e-bulletin sent July 2011. This highlighted recent local delayed HIV diagnoses, benefits of earlier testing and contact details of the sexual health clinic. We included an anonymised "ski-jump" graph showing ranked testing rates by practice, an invitation to GPs and practice managers to find out where their practice fell on the graph and the 2008 HIV testing guidelines, drawing attention to the table of indicator conditions.

Results Initial comparison of absolute numbers of tests and tests per head of practice population in GP practices in Cornwall during 2010 revealed large variation in testing rates between practices, from $<0.5$ tests per 1000 population to approximately 30 tests per 1000 population, with a total of 593 tests over 12 months. An interim analysis of HIV testing rates for the period April to September 2011 has shown an apparent increase in testing of $29 \%$ when projected for 12 months. Most practices showed increased rates of testing after the intervention.

Conclusion Our findings suggest that a simple targeted communication may result in increased testing in a low incident GP population. Monthly figures leading up to and following the intervention will be presented.

\section{P2 AUDIT OF CLINICAL STAGE AT PRESENTATION AND FACTORS CONTRIBUTING TO ANTIRETROVIRAL THERAPY DELAY, IN HIV PATIENTS IN RURAL KENYA}

doi:10.1136/sextrans-2012-050601c.2

R Hussey.* Forth Valley Royal Hospital

Background The 2010 WHO guideline, "Antiretroviral Therapy for HIV Infection in Adults and Adolescents" states that those with HIV and CD4 counts $\leq 350$ cells $/ \mathrm{mm}^{3}$, should start antiretroviral therapy (ART), regardless of symptoms. Those with WHO clinical stage 3/4 should start ART irrespective of CD4 count. In a small hospital north west of Nairobi, an HIV clinic, which is fully funded, provides ART to over 4000 patients. Patients are assessed on WHO clinical stage and CD4 count for ART eligibility.

Aim To audit a random sample of 100 patients attending the clinic and determine the WHO clinical stage at diagnosis, the number eligible for treatment at diagnosis and factors causing delay in ART initiation.

Method Retrospective audit of 100 case notes. Recording patient gender, age, date of diagnosis, WHO stage, CD4 count, length of time from diagnosis to ART initiation and reasons for delay of treatment. "Counselling" is compulsory pre-treatment, usually over 3 weeks. "Patient default" is defined as patient non-attendance.
Results $82 / 100$ patients were eligible for treatment at diagnosis WHO clinical stage at diagnosis: $1-26,2-17,3-34,4-5$. Length of time between diagnosis and treatment: $<28$ days: $21 / 82,>28$ days: 61/82, range: 4-861 days, mean: 102 days. Reasons for delay in those $>28$ days: TB diagnosis/treatment: 21 , counselling: 18 , patient default: 23 (see abstract P2 table 1).

Conclusion 82 of 100 HIV positive patients chosen at random were eligible for treatment at presentation, as per WHO guidelines. The majority of these patients were WHO stage 3 at presentation (41\%). Length of time from diagnosis to ART initiation varies greatly and this is likely multifactorial. A significant number of patients $(74 \%)$ did not commence ART within 4 weeks of diagnosis. The most common reason for this was patient non-attendance. In this setting, stigma attached to HIV diagnosis, extreme poverty and lack of education contribute to both late presentation and a delay in treatment initiation.

Abstract P2 Table 1 WHO clinical stage of HIV infection at diagnosis

\begin{tabular}{ll}
\hline WHO clinical stage at diagnosis & Number of patients \\
\hline 1 & 26 \\
2 & 17 \\
3 & 34 \\
4 & 5 \\
\hline
\end{tabular}

R Taylor,* M Pammi, P Venkatesan. Nottingham University Hospitals NHS Trust, Nottingham, UK

Background The BHIVA/BASHH/BIS UK Guidelines for HIV Testing (2008) state that all acute medical admissions in high prevalence areas, and all patients with specific clinical indicator conditions (CICs) should be offered an HIV test.

Aims As our Acute Trust serves a Primary Care Trust with a high prevalence of HIV (2.35/1000), we aimed to explore the attitudes towards HIV testing among hospital practitioners, the actual practice of testing and whether this reflected physician's stated attitudes

Methods An electronic survey was circulated to all doctors within the Trust. The survey explored respondents' experience, awareness, knowledge, training requirements and willingness to expand HIV testing. A separate paper-based questionnaire was sent to Clinical Leads in selected specialties to explore local testing policy.

Results 214 responses were received from the online-survey. $76 \%$ had experience in testing, 73\% felt they had necessary skills and $82 \%$ felt comfortable offering an HIV test. However, $45 \%$ had some concerns that their knowledge of HIV would be insufficient to answer patient questions. 99\% favoured expanding HIV testing. The specialty survey revealed that only $20 \%$ of specialties had any departmental policy on testing. For one-third of AIDS-defining CICs only selective testing was offered. $7 \%$ of CICs would never prompt a test, $7 \%$ would always prompt a test and $86 \%$ would prompt testing only in selective circumstances.

Conclusions Most doctors agreed with the importance of expanding HIV testing and felt they had the requisite skills. However, despite this, the practice of HIV testing varied and did not meet with UK Guidelines. If efforts to expand HIV testing in our Trust are to succeed, we need to continue to support and engage clinicians, to initially focus on facilitating strong departmental policies for AIDS-defining conditions prior to widening scope to include other testing strategies. 